\title{
Management of Chyle Leak in the Neck Following Thyroid Cancer Surgery: A Single Center Experience
}

\author{
${ }^{1}$ Anish Cherian, ${ }^{2}$ Pooja Ramakant, ${ }^{3}$ Mazhuvanchary Jacob Paul, ${ }^{4}$ Deepak Thomas Abraham
}

\begin{abstract}
Introduction: Surgery for thyroid cancers often necessitates a neck dissection. This is usually a safe procedure, but can be associated with complications. Chyle leak is one such complication, fortunately rare. There is a dearth of literature with regard to the management of chyle leak in the neck. We present a single center experience in the management of chyle leak in the neck, to improve the understanding of its management.
\end{abstract}

Materials and methods: A retrospective analysis of patients with thyroid cancer, managed between January 1st 2005 and December 31st 2011, in a single institution was performed. Among these, patients with chyle leak were identified. All pertinent data collected and results analyzed using STATA (v10).

Results: Three hundred and seventy-three/eight hundred and twenty-one (45.4\%) patients surgically managed for thyroid cancer underwent a neck dissection. Thoracic duct injury was recognized and managed intraoperatively in $20 / 373(5.4 \%)$ patients. The leak was prevented in the majority $(66.6 \%)$ of patients in whom a combination of methods were employed. $25 / 373(6.7 \%)$ patients were diagnosed and managed for chyle leak postoperatively. Seven patients required re-exploration. This included patients with low output chyle leaks who may have settled in a week to 10 days with conservative management. A combination of techniques was successful in the majority $(71.4 \%)$. The remaining patients were successfully managed conservatively.

Conclusion: We conclude that using a combination of methods to manage thoracic duct injury may be better than using a single modality alone. Early re-exploration was more economical and acceptable for a subset of our patients, as they come from long distances at personal cost.

Keywords: Thyroidectomy, Chyle leak, Thyroid cancer, Modified radical neck dissection.

How to cite this article: Cherian A, Ramakant P, Paul MJ, Abraham DT. Management of Chyle Leak in the Neck Following Thyroid Cancer Surgery: A Single Center Experience. World J Endoc Surg 2015;7(1):6-9.

Source of support: Nil

Conflict of interest: None

\section{INTRODUCTION}

Chyle leak is a rare complication following neck dissection for thyroid malignancy. Its incidence varies from

\footnotetext{
${ }^{1}$ Assistant Professor, ${ }^{2}$ Associate Professor, ${ }^{3,4}$ Professor

${ }^{1-4}$ Department of Endocrine Surgery, Christian Medical College Vellore, Tamil Nadu, India
}

Corresponding Author: Anish Cherian, Assistant Professor Department of Endocrine Surgery, Christian Medical College Vellore, Tamil Nadu, India, Phone: 04162282609, e-mail: anish@cmcvellore.ac.in
1 to $3 \%$, with an increased incidence following more radical surgical procedures. ${ }^{1-4}$ Patients who develop this complication are burdened by a protracted stay in hospital, escalating the cost of treatment and affecting their quality of life in the postoperative period. Literature on the management of chyle leak in the neck is scarce. We present our experience, over 7 years, in managing this complication in patients who are operated for thyroid cancer to improve the understanding of its management.

\section{MATERIALS AND METHODS}

Patients with thyroid cancer managed between 1st of January 2005 and 31st December 2011 ( $n=821)$, at a single center were identified from the electronic database. A retrospective chart review identified patients with a chyle leak. A detailed chart review of these patients was done and the results analyzed using STATA (v10).

\section{RESULTS}

A flow chart depicting the management of patients with a chyle leak is shown in Flow Chart 1.

- Chylous leak rate: Three hundred and seventy-three/ eight hundred and twenty-one (45.4\%) patients surgically treated for thyroid cancer had undergone a neck dissection. Among these, 25 (6.7\%) developed a chyle leak postoperatively. All the neck dissections performed were therapeutic.

- Intraoperative recognition: Thoracic duct injury was recognized and managed intraoperatively in 20/373 patients (Table 1). The management strategies employed (individually or in combination) include thoracic duct ligation, myoplasty and cyanoacrylate application. A flow chart depicting the management of the thoracic duct injury recognized intraoperatively is depicted in Flow Chart 2. Fisher exact test showed no significant difference $(p=0.179)$ between combination therapy and single modality therapy.

Eleven out of 20 patients (55\%) despite having managed the thoracic duct injury at the primary operation developed a chyle leak postoperatively. Three out of 11 (27\%) patients required re-exploration.

- Postoperative recognition: In the postoperative period, 25/373 patients were diagnosed and managed for chyle leak. Eleven out of 25 were patients with a 
persistent leak as mentioned above. In the remaining 14 patients, the thoracic duct injury was not identified at the primary operation and chyle leak was recognized only in the postoperative period. Ten of these patients could be managed conservatively while four required re-exploration.

- Re-exploration: Seven out of 25 patients with chyle leak in the postoperative period required re-exploration $(28 \%)$. The reason for re-exploration include high output chyle leak in two, failure to close in 1 to 2 weeks of conservative management in two and wound related complications (peridrain leak, collection of chyle and wound dehiscence) in the remaining three patients.

Three/seven patients were those who failed intraoperative management of thoracic duct injury at the primary operation. A combination of methods was used successfully during re-exploration in these

Flow Chart 1: Patients with chyle leak and their management

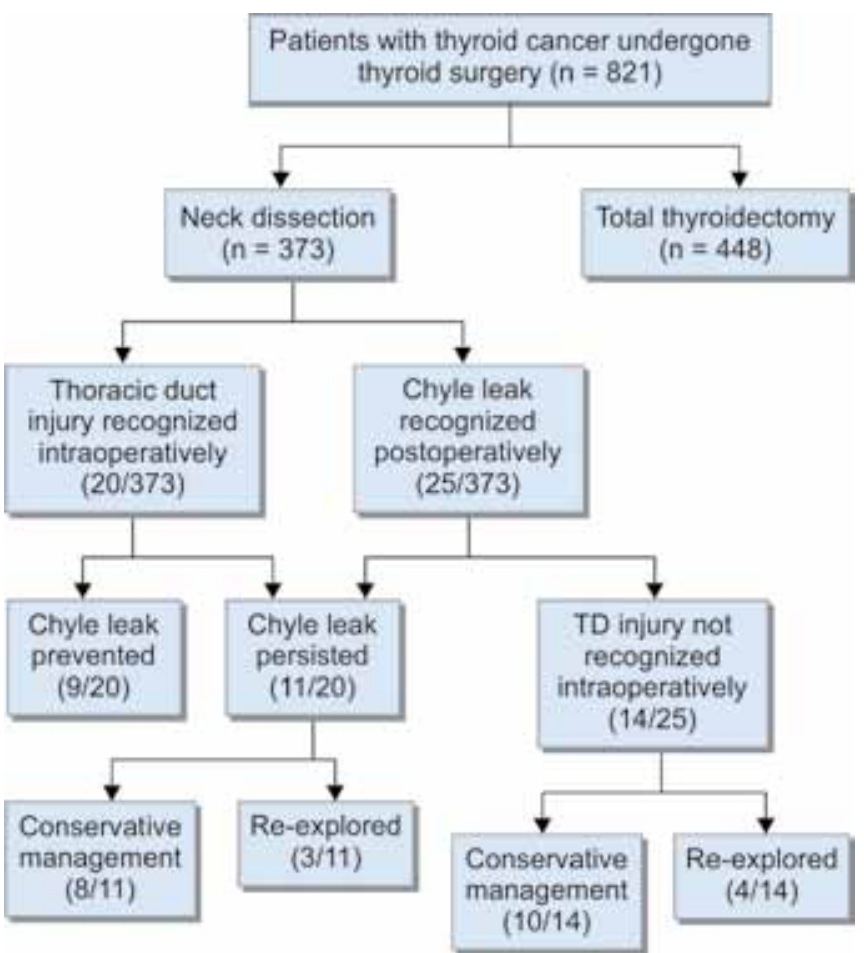

Flow Chart 2: The management of thoracic duct injury recognized intraoperatively

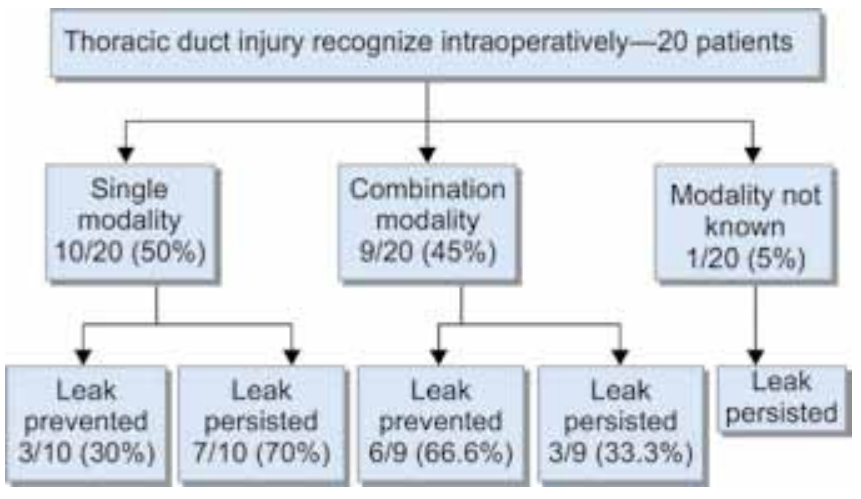

patients. The remaining four patients, the leak was identified only in the postoperative period. Among these four patients, combination of methods was used during re-exploration in two and a single modality in one. Both these patients had no further leak. The fourth patient the leak site was not identified, only an ectactic thoracic duct was seen. The wound was packed with (BIPP) bismuth, iodoform and paraffin paste. This patient was diagnosed with anaplastic thyroid cancer and was given palliative care only. This is the only patient who persisted to leak.

The duration to re-exploration varied among all the patients. Four patients were re-explored within a week. Two patients were re-explored after 2 weeks. The one patient with anaplastic carcinoma was re-explored after 30 days.

- Drain output: The drain output was low $(<500 \mathrm{ml} /$ day) in all except two patients.

- Right-sided leak: Six patients (24\%) had a right side chyle leak, five of whom healed spontaneously. One patient was given an initial trial of conservative management for 3 days. This patient had a high drain output $(>500 \mathrm{ml} /$ day), and required re-exploration. Suture ligating the thoracic duct and application of cyanoacrylate to the thoracic duct leak site was performed.

- Left-sided leak: Nineteen patients $(76 \%)$ had a leftsided chyle leak. Thirteen of these were successfully managed conservatively, while the remaining six patients failed initial conservative management and were re-explored. Five of the six patients re-explored had a low output chyle leak. All patients who healed spontaneously on conservative management did so within 2 weeks.

- Type of operation: Chyle leak is a known complication following lateral neck dissection. In our series as well, $24 / 25$ patients developed a chyle leak following a lateral neck dissection. One patient developed a leak following total thyroidectomy and therapeutic central compartment clearance alone.

\section{DISCUSSION}

\section{Chyle Leak Rate}

We report a high incidence of chylous leak from neck dissection for thyroid cancer compared to existing literature. A possible reason is that a higher proportion of our patients have an advanced disease as evidenced by the fact that $45.4 \%$ have undergone a therapeutic neck dissection.

\section{High Output vs Low Output}

The current definition of a low output chyle leak is a drain output less than $500 \mathrm{ml} /$ day. Twenty-three out of $25(92 \%)$ patients had a low output chyle leak. 
Table 1: Patients with thoracic duct injury identified intraoperatively, their management and outcomes

\begin{tabular}{clll}
\hline $\begin{array}{l}\text { Patient } \\
\text { no. }\end{array}$ & $\begin{array}{l}\text { Method of intraoperative management of thoracic duct injury (suture ligation of } \\
\text { thoracic duct/cyanoacrylate application/surgicel application/omohyoid myoplasty) }\end{array}$ & $\begin{array}{l}\text { Prevention of chyle } \\
\text { leak }(Y)^{*} /(\mathrm{N})^{\dagger}\end{array}$ & $\begin{array}{l}\text { Re-exploration } \\
\left(Y / N / N A^{\#}\right)\end{array}$ \\
\hline 1 & Suture ligation, cyanoacrylate and omohyoid myoplasty & $\mathrm{NA}$ \\
2 & Suture ligation, cyanoacrylate and omohyoid myoplasty & $\mathrm{Y}$ & $\mathrm{NA}$ \\
3 & Suture ligation, cyanoacrylate and omohyoid myoplasty & $\mathrm{Y}$ & $\mathrm{NA}$ \\
4 & Suture ligation, cyanoacrylate and omohyoid myoplasty & $\mathrm{N}$ & $\mathrm{NA}$ \\
5 & Suture ligation, omohyoid myoplasty & $\mathrm{Y}$ & $\mathrm{NA}$ \\
6 & Cyanoacrylate, omohyoid myoplasty & $\mathrm{Y}$ & $\mathrm{NA}$ \\
7 & Suture ligation & $\mathrm{Y}$ & $\mathrm{NA}$ \\
8 & Suture ligation & $\mathrm{Y}$ & $\mathrm{NA}$ \\
9 & Cyanoacrylate application & $\mathrm{Y}$ & $\mathrm{NA}$ \\
10 & Suture ligation, cyanoacrylate and omohyoid myoplasty & $\mathrm{N}$ & $\mathrm{N}$ \\
11 & Suture ligation, cyanoacrylate and omohyoid myoplasty & $\mathrm{N}$ & $\mathrm{N}$ \\
12 & Suture ligation, surgicel application & $\mathrm{N}$ & $\mathrm{N}$ \\
13 & Suture ligation & $\mathrm{N}$ & $\mathrm{N}$ \\
14 & Suture ligation & $\mathrm{N}$ & $\mathrm{N}$ \\
15 & Suture ligation & $\mathrm{N}$ & $\mathrm{N}$ \\
16 & Suture ligation & $\mathrm{N}$ & $\mathrm{N}$ \\
17 & Suture ligation & $\mathrm{N}$ & $\mathrm{N}$ \\
18 & Suture ligation & $\mathrm{N}$ & $\mathrm{N}$ \\
19 & Cyanoacrylate application & $\mathrm{N}$ & $\mathrm{N}$ \\
20 & Data not available & $\mathrm{N}$ & \\
\hline
\end{tabular}

${ }^{*} \mathrm{Y}$ : Yes; ${ }^{\dagger} \mathrm{N}$ : No; ${ }^{\#} \mathrm{NA}$ : Not applicable

\section{Left vs Right}

The majority of chyle leaks in the neck involve the left side as the thoracic duct terminates on the left side. ${ }^{5}$ There have been reports of chyle leak from the right side, up to $25 \%$ being reported..$^{6-8}$ This is due to injury to the right lymphatic duct. Our experience was similar with right sided chyle leak in six out of 25 patients (24\%). There are reports in literature of chyle leak following total thyroidectomy and central compartment lymph node dissection, $0.8 \%$ being reported. ${ }^{7}$ We also report low output leak from a central compartment dissection in one patient $(0.04 \%)$.

\section{MANAGEMENT}

\section{Intraoperative Recognition of Thoracic Duct Damage}

Various methods to identify if the thoracic duct has been injured during operation have been proposed. ${ }^{1}$ At our institution, we routinely perform the valsalva maneuver with direct inspection to check for chyle leak.

Once the site of the leak has been identified, the duct should be ligated using 3-0 or 4-0 prolene or the adjacent fascia plicated over the duct. ${ }^{9}$ Adjuncts to ligation include use of local and regional muscle flaps to aid in coverage of the site of injury. ${ }^{1}$ Other modalities used to increase likelihood of closure are fibrin/cyanoacrylate glue, sclerosing agents, such as OK 432 or tetracyclines (induce an inflammatory reaction in wound bed), and absorbable hemostat, such as surgicel and gelfoam. 1,2,6,10-12

From our experience using a combination of modalities to handle a thoracic duct injury seemed to be better than using a single modality alone. Though this was not statistically significant, the patient numbers being small a definite conclusion cannot be made. Even with control of all visible leakage sites, these patients are at a higher risk of postoperative chyle leaks. This may be due to unidentified injuries to other tributaries of the duct or additional termination of the duct. Majority of these usually settle on its own.

\section{Postoperative Recognition and Management}

In the postoperative period, chyle leaks may be low or high output. Low output chyle leaks are unlikely to have complications and may be managed conservatively. The aims of conservative management are to decrease chyle production, ensure adequate drainage, replace fluid and electrolyte and maintain/replete nutritional status.

Conservative management includes bed rest, maintaining suction drains placed during the operation and diet modified to an elemental diet with medium chain triglycerides (MCTs). ${ }^{1,2}$ If MCTs are not successful, then total parenteral nutrition (TPN) needs to be initiated. ${ }^{1,2}$ Other medical modalities have been tried, with varying results, to decrease chyle production. These include somatostatin and its analog octreotide, the pancreatic lipase inhibitor orlistat and etilefrine, a sympathomimetic amine. . $^{1,7,73-15}$

Conservative management in our series entailed only maintaining adequate drainage of the wound. We did not modify diet nor use TPN in any of our patients managed conservatively. 


\section{Re-exploration of Neck Wound with Repair of Chyle Leak}

Re-exploration of the neck wound with repair of site of chyle leak is indicated in those with wound complications or evidence of malnutrition/dyselectrolytemia, which is likely in patients who have a high output fistula (>500 ml/ day). Our patients with a low output chyle leak who failed to close in 1 to 2 weeks were re-explored early to reduce hospital stay and permit earlier return home. Further conservative management may have been successful in these patients. This strategy was more economical and acceptable to patients, many of whom come from long distances at personal cost. A combination of methods seems to be a better modality in treating chyle leaks during re-exploration as well.

\section{Newer Modalities of Management}

Percutaneous transabdominal cannulation of thoracic duct involves cannulating cisterna chyli or large lymphatic vessels transabdominally following pedal lymphangiography to delineate the lymphatic vessels. ${ }^{2,8}$ The damaged thoracic duct may be visualized, embolization coils and tissue adhesive may be placed. This is a time consuming procedure requiring multiple attempts, specialized personnel and equipment. It may also be associated with patient discomfort. Success rate has been reported in 45 to $70 \%$ of patients. ${ }^{8}$ We have no experience with this modality of management.

Thoracoscopic ligation of thoracic duct is another proposed method. This method had initially been advocated for chylothorax following esophagectomies and chest trauma. There have been a few case reports, where this method has been tried successfully to manage persistent/ high output chyle leaks in the neck. ${ }^{16,17}$ Here one identifies and ligates the thoracic duct in chest thoracoscopically. None of our patients in this cohort required thoracoscopic ligation of the thoracic duct to stop the chyle leak.

\section{CONCLUSION}

Chyle leak in the neck is a rare but potentially grave complication following neck operations. Our experience suggests that there may be a role for using a combination of methods to manage thoracic duct injury at primary operation rather than using a single modality alone. Majority of chyle leaks are low output. Early re-exploration for low output leaks may be considered in low/middle income countries to decrease the duration of hospital stay and in turn expenses. Left side is more commonly affected than the right side. Chyle leak may infrequently result following a central compartment clearance alone.

\section{REFERENCES}

1. Brennan PA, Blythe JN, Herd MK, Habib A, Anand R. The contemporary management of chyle leak following cervical thoracic duct damage. Br J Oral Maxillofac Surg 2012 Apr; 50(3):197-201.

2. Ilczyszyn A, Ridha H, Durrani AJ. Management of chyle leak post neck dissection: a case report and literature review. J Plastic Reconstruct Aesthet Surg 2011 Sep;64(9):e223-230.

3. Kinnaert P. Anatomical variations of the cervical portion of the thoracic duct in man. J Anat 1973 May;115(Pt 1):45-52.

4. Lee YS, Nam K-H, Chung WY, Chang H-S, Park CS. Postoperative complications of thyroid cancer in a single center experience. J Korean Med Sci 2010 Apr;25(4):541-545.

5. Lee LT, Poon CK, Cheng CS, Wong YK. Management of chylous leakage following neck dissection: case report. J Dent Sci 2008;3(1):57-61.

6. Nussenbaum B, Liu JH, Sinard RJ. Systematic management of chyle fistula: the Southwestern experience and review of the literature. Otolaryngology-Head and Neck Surgery 2000 Jan 1;122(1):31-38.

7. Rodier J-F, Volkmar P-P, Bodin F, Frigo S, Ciftci S, Dahlet C. Thoracic duct fistula after thyroid cancer surgery: towards a new treatment? Case Reports in Oncology 2011;4(2):255-259.

8. Scorza LB, Goldstein BJ, Mahraj RP. Modern management of chylous leak following head and neck surgery: a discussion of percutaneous lymphangiography-guided cannulation and embolization of the thoracic duct. Otolaryngologic Clinics of North America 2008 Dec;41(6):1231-1240.

9. Lucente FE, Diktaban T, Lawson W, Biller HF. Chyle fistula management. Otolaryngol Head Neck Surg 1981 Aug;89(4): 575-578.

10. Muthusami JC, Raj JP, Gladwin D, Gaikwad P, Sylvester S. Persistent chyle leak following radical neck dissection: a solution that can be the solution. Annals of the Royal College of Surgeons of England 2005 Sep 1;87(5):379.

11. Roh J-L, Yoon Y-H, Park CI. Chyle leakage in patients undergoing thyroidectomy plus central neck dissection for differentiated papillary thyroid carcinoma. Ann Surg Oncol 2008 Sep;15(9):2576-2580.

12. Metson R, Alessi D, Calcaterra TC. Tetracycline sclerotherapy for chylous fistula following neck dissection. Arch Otolaryngol Head Neck Surg 1986 Jun;112(6):651-653.

13. Nyquist GG, Hagr A, Sobol SE, Hier MP, Black MJ. Octreotide in the medical management of chyle fistula. Otolaryngology—Head and Neck Surgery 2003 Jun;128(6):910-911.

14. Rimensberger PC, Müller-Schenker B, Kalangos A, Beghetti M. Treatment of a persistent postoperative chylothorax with somatostatin. Ann Thorac Surg 1998 Jul;66(1):253-254.

15. Valentine CN, Barresi R, Prinz RA. Somatostatin analog treatment of a cervical thoracic duct fistula. Head Neck 2002 Aug;24(8):810-813.

16. Abdel-Galil K, Milton R, McCaul J. High output chyle leak after neck surgery: the role of video-assisted thoracoscopic surgery. Br J Oral Maxillofac Surg 2009 Sep;47(6):478-480.

17. Van Natta TL, Nguyen AT, Benharash P, French SW. Thoracoscopic thoracic duct ligation for persistent cervical chyle leak: utility of immediate pathologic confirmation. J Society of Laparoendoscopic Surgeons 2009;13(3):430. 